\title{
A Simple Iterative Method for Exact Solution of Systems of Linear Algebraic Equations
}

\author{
Albert Iskhakov ${ }^{1}$, Sergey Skovpen ${ }^{2}$ \\ ${ }^{1}$ Department of Innovation Development, 'VNIIEM Corporation' JC, Moscow, Russia. \\ ${ }^{2}$ Department of Ship Electricity and Automatics, Northern (Arctic) Federal University, Severodvinsk, Russia. \\ 1'asiskhakov@mail.ru, ${ }^{2}$ skovpensm@mail.ru
}

Abstract: We present for the first time a theorem (with proof) of solving a system of linear algebraic equations (SLAE) in a finite number of iterations with a stationary matrix. The system is transformed such that the spectrum of the matrix to be unit. Splitting the matrix gives a nilpotent matrix of the iteration system that generates an exact solution of SLAE for a number of iterations not exceeding the dimension of the system.

Keywords: Simple Iterative Method, System of Linear Algebraic Equations (SLAE), Exact Solution, Nilpotent Matrix, Unipotent Matrix, Stationary Matrix, Finite Iterative Process

\section{INTRODUCTION}

The mathematical community considers that a simple iterative method expressed as a linear difference equation for solving a system of linear algebraic equations (SLAE) is approximate because it does not give the exact answer for a finite number of iterations. The statements on this matter can be found in [1-4]. However, in control theory, for a linear discrete system described by the same equation, a method for transferring the system to a given state in a finite number of steps has been known for a long time [5-7].

A method to findthe exact solution of SLAE in a finite number of iterations is reported for the first time. Some points of the methodare stated in [8-10], in particular, the iterative equations for a second-order SLAE represented in analytical form are given in [10]. Here we present a theorem and prove it.

\section{THEOREM AND PROOF}

Theorem: To find a solution of SLAE

$$
A x=b,
$$

Where $x$ and $b$ are the unknown and known vectors of $k$ size, respectively, $A$ is a square nonsingular matrix of $k$ size, in a finite number of iterations, it is necessary and sufficient to reduce (1) to the form

$$
E x=c \text {, }
$$

where $E$ is a unipotent matrix with unit spectrum. Then the iterative equation

$$
x_{n+1}=N x_{n}+c
$$

where $n=0,1,2, \ldots, E=I-N$, I is the identity matrix, for an arbitrary initial vector $x_{\sigma^{\prime}}$ generates the exact solution (excluding round-off errors)

$$
x=A^{-1} b=E^{-1} c
$$

no more than for $m \leq k$ iterations. 
Proof. The matrix $E$ is split into the identity matrix $I$ and the nilpotent matrix $N$ with the intrinsic property

$$
N^{k}=0 .
$$

A non-unipotent matrix does not form a nilpotent one, and, with $c=0$, the solution to (3) given by

$$
x_{n}=N^{n} x_{0}
$$

depends on $x_{0}$. This proves the necessity.

The sufficiency follows from the solution of (3) by using (5) for $n=k$ :

$$
x_{k}=N^{k} x_{0}+\left(I+N+\ldots+N^{k-1}\right) c=(I-N)^{-1} c=x .
$$

\section{Remark1}

The simple iterative method is approximate due to the extension of the condition $\operatorname{det} A \neq 0$ to the matrix of the iteration equation, as follows from control theory and this statement is confirmed here, but this is not necessary.

\section{Remark 2}

The transformation of the difference equation to the form with nilpotent matrix, used in control theory, with $c=0$, results in to zero in a finite number of steps, and, with $c \neq 0$, it gives the shifted vector (as it was named in [7]) instead of the solution.

\section{Remark 3}

The specified spectrum is formed by changing the elements of a row of the matrix $A$ itself, while in control theory, a row of the elements of the characteristic polynomial of the Frobenius matrix is changed, which is obtained by the transformation of $A$.

\section{Remark 4}

There are values $x_{0}$ with which the number $m$ equals to $1,2, \ldots,(k-1)$, in particular, $m=k-1$ for $x_{0}=c$.

\section{CONCLUSION}

Aniterative method for exact solution of a SLAE in a finite number of steps has the computational procedure of iterative methods, in the conventional sense, like the Jacobi and Seidel methods, can be called a finite-iterative method. As in control theory, it is based on setting a unit spectrum for the SLAE matrix or a zero spectrum for the matrix of the iteration equation $[8,9]$. The main difference is possibility to solve not only a homogeneous equation, but also a non-homogeneous one.

The transformation of a SLAE to the form with a unipotent matrix is achieved, according to the control theory terminology, by the feedback on the first difference of the desired vector, which is mathematically a special case of the canonical form of a one-step two-layer iterative method. Its estimation is still ahead, but the already obtained results of comparison using the example of a third-order SLAE with such a non-stationary iterative method as the method of conjugate gradients demonstrate higher accuracy.

For the sake of clarity, the application of the method in detail will be considered separately on the example of a second-order SLAE and illustrated by obtaining Kramer's formulas in analytical form after one or two iterations. 


\section{REFERENCES}

1. Fadeev, D.K. and Fadeeva, V.N. (1963) Computational Methods of Linear Algebra. Freeman, San Francisco, CA.

2. Strang, G. (1976) Linear Algebra and Its Applications. Academic Press, New York.

3. Samarskii, A.A. and Nikolaev, E.S. (1978) Methods for Solving the Grid Equations. Nauka, Moscow (in Russian).

4. Demmel, W.D. (1997) Applied Numerical Linear Algebra. Society for Industrial and Applied Mathematics, Philadelphia.

5. Kwakernaak, H. and Sivan, R. (1972) Linear Optimal Control Systems. Wiley-Interscience, New York.

6. Isermann, R. (1981) Digital Control Systems. Springer-Verlag, Berlin, Heidelberg.

7. Pervozvanskii, A.A. (1987) Course on Automatic Control Theory. Nauka, Moscow (in Russian).

8. Iskhakov A., Pospelov V., Skovpen S. Non-Frobenius Spectrum-Transformation Method.Applied Mathematics, 2012, Vol. 3, No. 1, pp. 1471-1479.doi: 10.4236/am.2012.330206

9. Iskhakov A., Skovpen S. A Direct Transformation of a Matrix Spectrum. Advances in Linear Algebra \& Matrix Theory, 2015, Vol. 5, No. 3, pp. 109-128. doi: 10.4236/alamt.2015.53011

10. Iskhakov A., Skovpen S. Exact Solution of a Linear Difference Equation in a Finite Number of Steps. Applied Mathematics, 2018, No. 9, pp. 287-290.doi: 10.4236/am.2018.93022

Citation: Albert Iskhakov, Sergey Skovpen, "A Simple Iterative Method for Exact Solution of Systems of Linear Algebraic Equations. American Research Journal of Mathematics; vol 5, no. 1, 2019, pp. 1-3.

Copyright (C) 2019 Albert Iskhakov, Sergey Skovpen. This is an open access article distributed under the Creative Commons Attribution License, which permits unrestricted use, distribution, and reproduction in any medium, provided the original work is properly cited. 